\title{
MODELING OF LINEAR ISENTROPIC FLOW SYSTEMS
}

\author{
Athan D. Sarantopoulos \& Tom T. Hartley \\ Department of Electrical Engineering \\ The University of Akron \\ Akron, Ohio 44325-3904 \\ e-mait.Sarantopoulos@uakron.edu
}

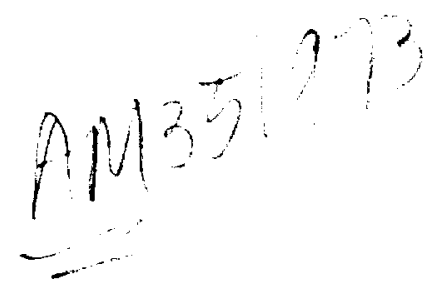

\begin{abstract}
A modeling approach for linear isentropic flow systems based on the quasi-one dimensional Euler equations of non-viscous, compressible flow is presented. Such systems are representative of certain high speed propulsion systems. Accurate models useful in control system studies are developed. A supersonic inlet is considered and the resulting set of partial differential equations with boundary conditions is solved for a linear transfer matrix using Laplace transforms.
\end{abstract}

\section{INTRODUCTION}

High speed propulsion systems, such as supersonic inlets, can be described by the quasione dimensional set of Euler equations which represent non-viscous compressible flow. Quasione dimensional flow occurs in a channel with a small rate of change of cross section (see Figure 1 below) or between nearly parallel streamlines in which case the velocity components normal to the mean flow direction are small compared to the components parallel to the flow direction.

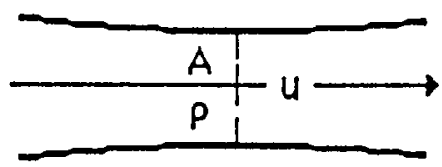

Figure 1. A Stream Tube.

The resulting set of equations is nonlinear and includes equations for conservation of mass, conservation of momentum, and conservation of energy. Usually, any solution to this set of equations requires computational fluid dynamics (CFD). However, if it is assumed that any perturbation in the flow field is small, a linearized set of equations results. Furthermore, if it is also assumed that any energy wave is insignificant, then the conservation of energy equation can be eliminated from the set. This sifuation is called isentropic flow, as the entropy remains constant in the flow field.
The following linear isentropic flow equations now result:

$$
\begin{aligned}
& \frac{\partial u}{\partial t}+\bar{u} \frac{\partial u}{\partial x}+\frac{c^{2}}{\bar{\rho}} \frac{\partial \rho}{\partial x}=f_{u} \\
& \frac{\partial \rho}{\partial t}+\bar{p} \frac{\partial u}{\partial x}+\bar{u} \frac{\partial \rho}{\partial x}=f_{p}
\end{aligned}
$$

where $u$ is the flow velocity, $\rho$ is the density of the medium, the f's are spatially distributed inputs, $c$ is the speed of sound, and the barred variables represent the steady state values around which these equations were linearized.

\section{BVP PROBLEM FOR A SUPERSONIC INLET}

To apply the linear isentropic flow equations to a real system, boundary conditions are necessary. For a specific solution, a supersonic inlet is considered. Here the downstream boundary is simply reflective. In order to represent compressor or combustor signals, a perturbation input $f_{d s}$ is also included in the downstream boundary. On the other hand, the upstream boundary is considered to be the normal shock usually located near the throat of a supersonic inlet. Furthermore, to represent perturbations in the free stream atmosphere, an upstream input $f_{u s}$ is included. Symbolically, these conditions are

$$
\rho_{d s}=R u_{d s}+f_{d s}, \quad u_{u s}=Z_{u s}(s) \rho_{u s}+f_{u s} \text {, }
$$

where $R$ is a constant representing the reflective boundary and $Z_{u s}=Z_{u s}(s)$ is a lumped dynamic representing the shock behavior (see Figure 2).

Using zero initial conditions and Laplace. transforming the given system yields:

$$
\begin{aligned}
& \text { s } u(s, x)+\bar{u} \frac{\partial u(s, x)}{\partial x}+\frac{c^{2}}{\bar{\rho}} \frac{\partial \rho(s, x)}{\partial x}=f_{u} \\
& s \rho(s, x)+\bar{\rho} \frac{\partial u(s, x)}{\partial x}+\bar{u} \frac{\partial \rho(s, x)}{\partial x}=f_{p}
\end{aligned}
$$

If $f_{u}=f_{p}=0$, then using matrix notation the above system becomes,

where,

$$
A \frac{\partial \underline{u}(s, x)}{\partial x}+B \underline{u}(s, x)=0
$$

$$
A=\left[\begin{array}{ll}
\bar{u} & \frac{c^{2}}{\bar{\rho}} \\
\bar{\rho} & \bar{u}
\end{array}\right], \underline{u}=\left[\begin{array}{l}
u(s, x) \\
\rho(s, x)
\end{array}\right], B=\left[\begin{array}{ll}
s & 0 \\
0 & s
\end{array}\right]
$$


Since, matrix $A$ is nonsingular, the system can be rewritten as,

$$
\frac{\partial \underline{u}(s, x)}{\partial x}+A^{-1} B \underline{u}(s, x)=0
$$

The boundary conditions for this system, using the notation of reference 1, can be put in the following form,

or,

$$
M(s) Q(s, 0)+N(s) Q(s, L)=Z(s)
$$

where,

$$
M(s) Q_{u s}+N(s) Q_{d s}=Z(s)
$$

and,

$$
M(s)=\left[\begin{array}{cc}
1 & -Z_{u s} \\
0 & 0
\end{array}\right], N(s)=\left[\begin{array}{cc}
0 & 0 \\
-R & 1
\end{array}\right]
$$

$$
Q(s, x)=\left[\begin{array}{l}
u(s, x) \\
p(s, x)
\end{array}\right], Z(s)=\left[\begin{array}{l}
f_{u s} \\
f_{d s}
\end{array}\right]
$$

Note that since $Z(s) \neq 0$, the termination is said 10 be "nonhomogeneous." The solution of the system is $\underline{u}(s, x)=e^{-A^{-1} B x} \underline{u}(s, 0) \equiv H(s, x) \underline{u}(s, 0) \equiv H(s, x) \underline{u_{u s}}$ where, the open loop or "free space" transfer function matrix $H(s, x)$ is given by

$H(s, x)=\exp \left(\frac{\bar{u} s x}{c^{2}-\bar{u}^{2}}\right)\left[\begin{array}{cc}\cosh \left(\frac{c s x}{c^{2}-\bar{u}^{2}}\right) & \frac{-c}{\bar{p}} \sinh \left(\frac{c s x}{c^{2}-\bar{u}^{2}}\right) \\ \frac{-\vec{\rho}}{c} \sinh \left(\frac{c s x}{c^{2}-\vec{u}^{2}}\right) & \cosh \left(\frac{c s x}{c^{2} \cdot \vec{u}^{2}}\right)\end{array}\right]$

The existence of hyperbolic functions in $H(s, x)$ is an indication that the resulting model is a complicated iransfer function having an infinite number of poles and zeros, characteristic of disiributed parameter systems. The transfer function matrix from $Z(s)$ to $Q(s, x)$ can be shown to be given by

$K(s, x)=H(s, x)[M(s)+N(s) H(s, L)]^{-1}$ Thus, calculation of $K(s, x)$ requires first computing $H(s, L)$. From $H(s, x)$, given above, it follows that,

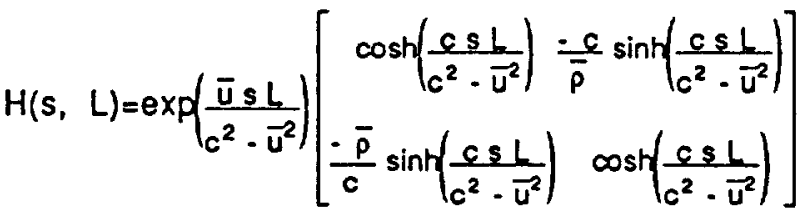

Furthermore, substituting $M(s), N(s)$, and $H(s, L)$ into $K(s, x)$ one obtains the following expressions.

$$
\begin{aligned}
& {[M(s)+N(s) H(s, L)]^{-1}} \\
& =\left\{\left[\begin{array}{cc}
1 & -Z_{u s} \\
0 & 0
\end{array}\right]+\left[\begin{array}{cc}
0 & 0 \\
-R & 1
\end{array}\right]\left[\begin{array}{ll}
H_{11}(s, L) & H_{12}(s, L) \\
H_{21}(s, L) & H_{22}(s, L)
\end{array}\right]\right]^{-1}
\end{aligned}
$$

$$
\begin{aligned}
& =\left[\begin{array}{cc}
1 & -Z_{u s} \\
-R H_{11}(s, L)+H_{21}(s, L) & -R H_{12}(s, L)+H_{22}(s, L)
\end{array}\right]^{-1} \\
& =\frac{1}{H_{22}(s, L) \cdot R H_{12}(s, L) \cdot Z_{u s} R H_{11}(s, L)+Z_{u s} H_{21}(s, L)} \\
& \cdot\left[\begin{array}{cc}
-R H_{12}(s, L)+H_{22}(s, L) & Z_{u s} \\
R H_{11}(s, L)-H_{21}(s, L) & 1
\end{array}\right]
\end{aligned}
$$

Thus, the transfer function matrix $K(s, x)$ is given by, $K(s, x)=H(s, x)[M(s)+N(s) H(s, L)]^{-1} \equiv K_{0}\left[\begin{array}{ll}K_{1} & K_{2} \\ K_{3} & K_{4}\end{array}\right]$ where, the quantities $K_{0}, K_{1}, K_{2}, K_{3}$, and $K_{4}$ are given next.

$$
\begin{aligned}
& K_{0}=\frac{\exp \left(\frac{\bar{u} s x}{c^{2}-\bar{u}^{2}}\right)}{H_{22}(s, L) \cdot R H_{12}(s, L) \cdot Z_{u s} R H_{11}(s, L)+Z_{u s} H_{21}(s, L)} \\
& =\frac{\exp \left[\frac{\bar{u} s(x-L)}{c^{2} \cdot \bar{u}^{2}}\right]}{\left(1-Z_{u s} R\right) \cosh \left(\frac{c s L}{c^{2}-\bar{u}^{2}}\right)+\left(\frac{R c}{\bar{\rho}} \cdot Z_{u s} \frac{\bar{\rho}}{c}\right) \sinh \left(\frac{c s L}{c^{2}-\bar{u}^{2}}\right)} \\
& K_{1}=\left\{\cosh \left(\frac{c s x}{c^{2}-\bar{u}^{2}}\right)\left[R \frac{c}{\bar{\rho}} \sinh \left(\frac{c s L}{c^{2}-\bar{u}^{2}}\right)+\cosh \left(\frac{c s L}{c^{2}-\bar{u}^{2}}\right)\right]-\frac{c}{\bar{\rho}} \cdot\right. \\
& \cdot \sinh \left(\frac{c s x}{c^{2}-\bar{u}^{2}}\right)\left[R \cosh \left(\frac{c s L}{c^{2} \cdot \bar{u}^{2}}\right)+\frac{\bar{\rho}}{c} \sinh \left(\frac{c s L}{c^{2}-\bar{u}^{2}}\right)\right] \int \exp \left(\frac{\bar{u} s L}{c^{2}-\bar{u}^{2}}\right) \\
& =\left\{\cosh \left(\frac{c s(x-L)}{c^{2}-\bar{u}^{2}}\right) \cdot \frac{R c}{\bar{\rho}} \sinh \left(\frac{c s(x \cdot L)}{c^{2}-\bar{u}^{2}}\right)\right\} \exp \left(\frac{\bar{u} s L}{c^{2}-\bar{u}^{2}}\right)
\end{aligned}
$$$$
K_{2}=Z_{u s} \cosh \left(\frac{c s x}{c^{2}-\bar{u}^{2}}\right) \cdot \frac{c}{\bar{\rho}} \sinh \left(\frac{c s x}{c^{2} \cdot \bar{u}^{2}}\right)
$$$$
K_{3}=\left(\frac{\bar{p}}{c} \sinh \left(\frac{c s x}{c^{2}-\bar{u}^{2}}\right)\left[\frac{B c}{\bar{\rho}} \sinh \left(\frac{c s L}{c^{2}-\bar{u}^{2}}\right)+\cosh \left(\frac{c s L}{c^{2}-\bar{u}^{2}}\right)\right]+\right.
$$$$
\left.\cosh \left(\frac{c s x}{c^{2} \cdot \bar{u}^{2}}\right)\left[R \cosh \left(\frac{c s L}{c^{2} \cdot \bar{u}^{2}}\right)+\frac{\bar{e}}{c} \sinh \left(\frac{c s L}{c^{2} \cdot \bar{u}^{2}}\right)\right]\right] \exp \left(\frac{\bar{u} s L}{c^{2} \cdot \bar{u}^{2}}\right)
$$$$
=\left\{R \cosh \left(\frac{c s(x-L)}{c^{2} \cdot \bar{u}^{2}}\right)-\frac{\bar{\rho}}{c} \sinh \left(\frac{c s(x-L)}{c^{2} \cdot \bar{u}^{2}}\right)\right\} \exp \left(\frac{\bar{u} s L}{c^{2}-\bar{u}^{2}}\right)
$$

$$
K_{A}=\cosh \left(\frac{c s x}{c^{2}-\bar{u}^{2}}\right)-\frac{\bar{c}}{c} Z_{u s} \sinh \left(\frac{c s x}{c^{2} \cdot \bar{u}^{2}}\right)
$$

Finally, the bounded system is as follows:

$$
Q(s, x)=\left[\begin{array}{l}
u(s, x) \\
\rho(s, x)
\end{array}\right]=K(s, x)\left[\begin{array}{l}
f_{u s} \\
f_{d s}
\end{array}\right]=K(s, x) Z(s)
$$


$K(s, x)$ is referred to as the transfer function matrix for the given boundary conditions and is given by,

$$
\begin{aligned}
& K(s, x)=\left[\begin{array}{ll}
K_{11} & K_{12} \\
K_{21} & K_{22}
\end{array}\right]= \\
& \frac{\exp \left[\frac{\bar{u} s(x-L)}{c^{2}-\bar{u}^{2}}\right]}{\left(1-Z_{u s} R\right) \cosh \left(\frac{c s L}{c^{2}-\bar{u}^{2}}\right)+\left(\frac{R c}{\bar{\rho}} Z_{u s} \frac{\bar{\rho}}{c}\right) \sinh \left(\frac{c s L}{c^{2}-\bar{u}^{2}}\right)} \text {. } \\
& {\left[\begin{array}{l}
\left\{\cosh \left(\frac{c s(x-L)}{c^{2} \cdot \bar{u}^{2}}\right)-\frac{R c}{\bar{p}} \sinh \left(\frac{c s(x-L)}{c^{2}-\bar{u}^{2}}\right)\right\} \exp \left(\frac{\bar{u} s L}{c^{2}-\bar{u}^{2}}\right) \\
\left\{R \cosh \left(\frac{c s(x-L)}{c^{2}-\bar{u}^{2}}\right)-\frac{\bar{P}}{c} \sinh \left(\frac{c s(x-L)}{c^{2}-\bar{u}^{2}}\right)\right) \exp \left(\frac{\bar{u} s L}{c^{2}-\bar{u}^{2}}\right)
\end{array}\right.} \\
& \vdots Z_{u s} \cosh \left(\frac{c s x}{c^{2}-\bar{u}^{2}}\right)-\frac{c}{\bar{\rho}} \sinh \left(\frac{c s x}{c^{2} \cdot \bar{u}^{2}}\right) \\
& \left.\cosh \left(\frac{c s x}{c^{2} \cdot \bar{u}^{2}}\right)-\frac{\bar{p}}{c} Z_{u s} \sinh \left(\frac{c s x}{c^{2} \cdot \bar{u}^{2}}\right)\right)
\end{aligned}
$$

It is interesting to note that every term in $K(s, x)$ is a ratio of entire functions. This is true because the quantity $M(s)+N(s) H(s, L)$ as well as its adjoint is a sum of entire functions.

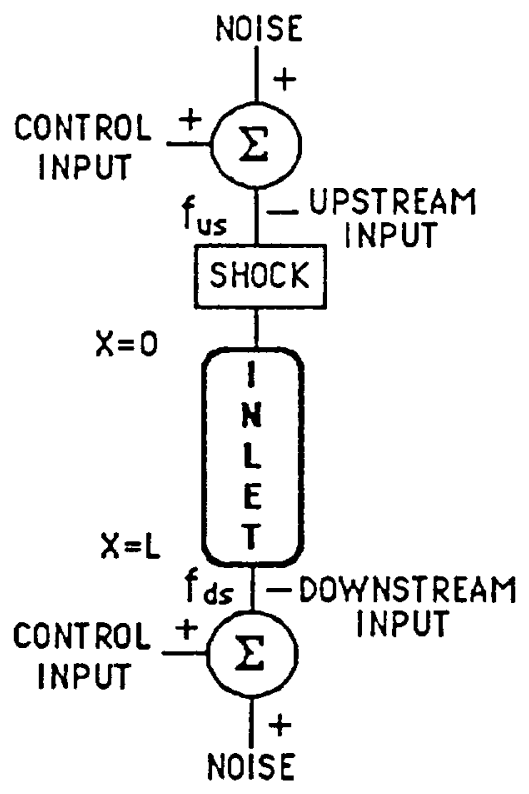

Figure 2. A Supersonic Inlet System.

\section{APPLICATION}

From the previous section, it can be seen that $Q(s, x)$ is the output vector of the closed loop system when its input is the vector

$$
Z(s)=\left[\begin{array}{ll}
f_{u s} & f_{d s}
\end{array}\right]^{\top}
$$

$f_{u s}$ and $f_{d s}$ will be assumed to be two noise corrupted control inputs to the supersonic inlet system. This situation is illustrated in Figure 2.

It should be noted that the system transfer function $K(s, x)$ can be more complicated than it appears since $Z_{\text {us }}$ is a lumped dynamic system representing the shock behavior. In general, this can be a ratio of two polynomials having $m$ zeros and $n$ poles. The corresponding mathematical model for Zus would then be as follows:

$$
Z_{u s}(s)=\frac{\sum_{l=0}^{m} b_{1} s^{m-1}}{\sum_{j=0}^{n} a_{j} s^{n-1}}=\frac{b_{0} s^{m}+b_{1} s^{m-1}+\ldots+b_{m-1} s+b_{m}}{a_{0} s^{n}+a_{1} s^{n-1}+\ldots+a_{n-1} s+a_{n}}
$$

where, it is usually assumed that $a_{0} \neq 0$, and $b_{0} \neq 0$. However, it has been shown experimentally (see references 2 and 3) that $Z_{u s}(s)$ can be modeled as a first order system having a last lag. This suggests that $\mathrm{Zus}_{\mathrm{u}}(\mathrm{s})$ takes the following form:

$$
Z_{u s}(s)=\frac{Z_{0}}{s-p}
$$

Using the data shown in Table 1, which can be obtained from reference 4, one can compute the frequency response of the closed loop system at $x=0$, which will give an approximate response for the shock.

Table 1. Supersonic Inlet Data

\begin{tabular}{|c|c|}
\hline $\bar{u}$ & 574 \\
\hline $\bar{p}$ & 0.00136 \\
\hline$c$ & 1135 \\
\hline$L$ & 2.57 \\
\hline
\end{tabular}

The shock transfer function is assumed to be

$$
Z_{u s}(s)=\frac{10^{6}}{s+300}
$$

and the reflection parameter, $\mathrm{A}$, is chosen to be $R=6 \cdot 10^{-7}$. At this point, the values for $R$ and $Z_{0}$ are roughly chosen so that the magnitude of the reflections from the boundaries are approximately unity. Further research to develop a more accurate technique for determining $R$ and $Z_{0}$ as well as better shock models is currently being conducled.

Using the above data the response shown in Figure 3 is obtained. Note that only the $K_{12}(s, 0)$ term is shown. For comparison purposes the frequency response of this system computed in reference 4 is superimposed on the frequency response of $K_{12}(s, 0)$.

Furthermore Figure 4 illustrates a threedimensional plot of $\log \left|K_{12}(s, 0)\right|$ versus $s$. The peaks in the plot correspond to the pole locations of $\mathrm{K}_{12}(s, 0)$. 


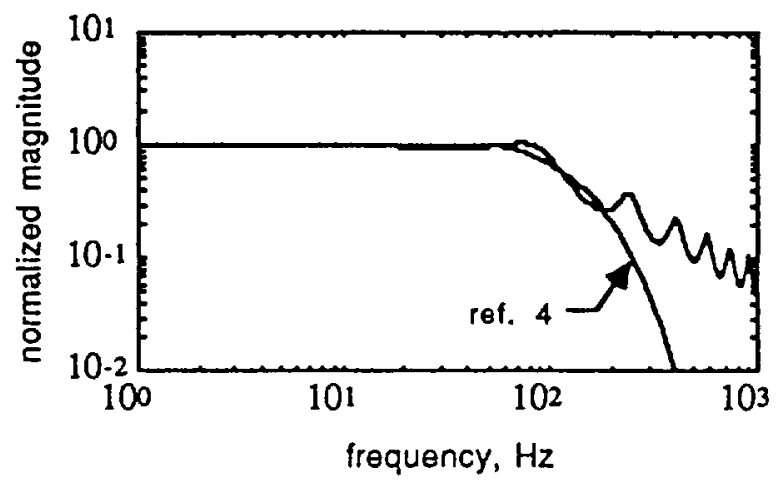

Figure 3. Magnitude Response for $\left|K_{12}(s, 0)\right|$.

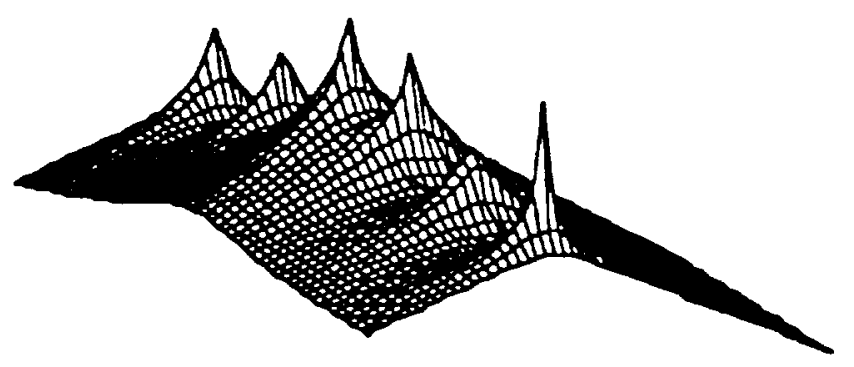

Figure 4. $\log \left|K_{12}(s, 0)\right|$ versus $s$.

To better understand the location of the poles, a contour plot is also constructed in Figure 5. Closed curves represent points in the $s$ plane that yield the same magnitude in the frequency response. Note that all the poles seem to lie in a strip approximately between $s=-150$ and $s=-350$ and they are symmetrical about the real axis.

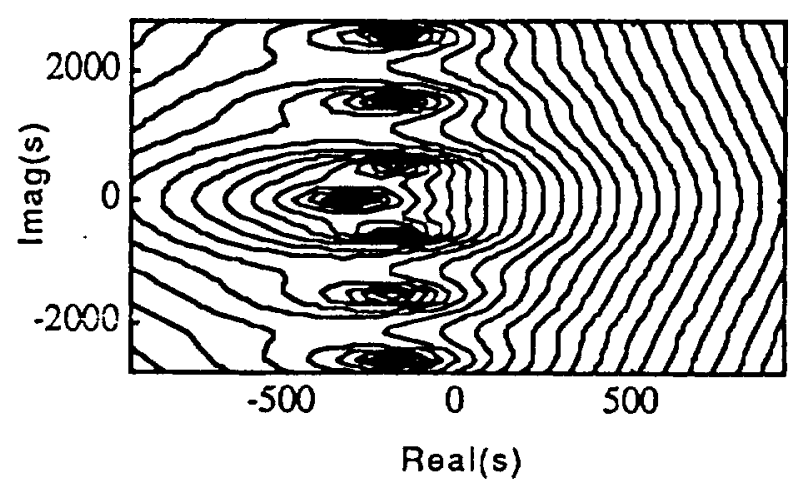

Figure 5. A Contour Plot for $\left|K_{12}(s, 0)\right|$.

\section{CONCLUSIONS}

The resulting model is very useful to the control engineer. It can provide quick fast insight about this very complicated system. The solution obtained here is in a compact form and one can get the answer quickly. In fact, a MATLAB program has been developed to compute frequency responses for various conditions and shock models, which are in turn useful for control system design using root locus or frequency domain techniques.

The analytical solution obtained here is an exact solution of the boundary value problem as opposed to the approximate solutions presented by Cole and Willoh in references 2 and 3 . The solution is good as long as the conditions for a small perturbation model are met. However, to obtain a better model, the energy equation should be added to the set.

Finally, it is noted that the free space system function matrix given in this paper is very similar to that of an electrical transmission line system. In fact, both the inlet and the transmission line systems are in a sense identical for $\bar{u}=0$.

\section{ACKNOWLEDGEMENT}

This work was partially supported by the Advanced Control Technology Branch of NASA Lewis Research Center via grant NAG 3-904.

\section{REFERENCES}

[1] R. J. Schwarz and B. Friedland,: Linear Systems, McGraw-Hill, New York, 1965.

[2] R. G. Willow, "A mathematical analysis of supersonic inlet dynamics," NASA TN D. 4969, 1968.

[3] G. L. Cole and R. G. Willow, "Analysis of the dynamic response of a supersonic inlet to flow-field perturbations upstream of the normal shock," NASA TN D-7839, 1975.

[4] A. Chicatelli, "Methods for developing linear reduced models of internal flow propulsion systems," Masters Thesis, University of Akron, 1990.

Presented to the IEEE International Conference on Systems Engineering, Wright State University, Dayton, OH, August 1-3 1991. 